\title{
Modeling of Human Uveal Melanoma in Zebrafish Xenograft Embryos
}

\author{
Wietske van der Ent, ${ }^{1,2}$ Claudia Burrello, ${ }^{1}$ Amina F. A. S. Teunisse, ${ }^{3}$ Bruce R. Ksander, ${ }^{4}$ \\ Pieter A. van der Velden, ${ }^{5}$ Martine J. Jager, ${ }^{5}$ Aart G. Jochemsen, ${ }^{3}$ and B. Ewa Snaar-Jagalska ${ }^{1}$
}

${ }^{1}$ Institute of Biology, Leiden University, Leiden, The Netherlands

${ }^{2}$ Department of Pathology, Leiden University Medical Center, Leiden, The Netherlands

${ }^{3}$ Department of Molecular Cell Biology, Leiden University Medical Center, Leiden, The Netherlands

${ }^{4}$ Department of Ophthalmology, Massachusetts Eye and Ear Infirmary, Schepens Eye Research Institute, Harvard Medical School, Boston, Massachusetts, United States

${ }^{5}$ Department of Ophthalmology, Leiden University Medical Center, Leiden, The Netherlands

Correspondence: Aart G. Jochemsen, Leiden University Medical Center, P.O. Box 9600, 2300 RC Leiden, The Netherlands;

a.g.jochemsen@lumc.nl.

B. Ewa Snaar-Jagalska, Einsteinweg 55, 2333 CC Leiden, The Netherlands;

b.e.snaar-jagalska@biology.leidenuniv.nl. WvdE and CB contributed equally to the work presented here and should therefore be regarded as equivalent authors.

Submitted: July 10, 2014

Accepted: September 11, 2014

Citation: van der Ent W, Burrello C, Teunisse AFAS, et al. Modeling of human uveal melanoma in zebrafish xenograft embryos. Invest Ophthalmol Vis Sci. 2014;55:6612-6622. DOI:10.1167/iovs.14-15202
Purpose. Uveal melanoma (UM) is fatal in up to $50 \%$ of patients because of liver metastases that are refractory to therapies currently available. While murine xenograft models for human uveal melanoma are available, they have limited utility for screening large compound libraries in drug discovery studies. Therefore, new robust preclinical models are needed that can efficiently evaluate drug efficacy for treatment of this malignancy.

Methods. Uveal melanoma cell lines generated from primary tumors (92.1, Mel270) and metastases (OMM2.3, OMM2.5, OMM1) were injected into the yolk of 2-day-old zebrafish embryos. After 6 days, proliferation and active migration was quantified via automated confocal image analysis. To determine the suitability of this xenotransplantation model for drug testing, drugs with three different activities (dasatinib, quisinostat, and MLN-4924) were added to the water of uveal melanoma-engrafted embryos.

REsults. All tested UM cell lines proliferated and migrated in the embryos; significant differences could be discerned between cell lines: Cells derived from metastases showed more migration and proliferation than cells derived from the primary tumors, and provided preclinical models for drug testing. Addition of the Src-inhibitor dasatinib in the water of engrafted embryos reduced proliferation and migration of high Src-expressing 92.1 cells, but did not affect low Src-expressing metastatic OMM2.3 cells. Two experimental anticancer drugs, quisinostat (a histone deacetylase inhibitor) and MLN-4924 (neddylation pathway inhibitor), blocked migration and proliferation of 92.1 and OMM2.3.

Conclusions. We established a zebrafish xenograft model of human uveal melanoma with demonstrated applicability for screening large libraries of compounds in drug discovery studies.

Keywords: uveal melanoma, xenotransplantation, zebrafish, drug treatment
$\mathrm{U}$ veal melanoma (UM) is the most common primary intraocular malignancy in adults and accounts for $5 \%$ of all melanomas. ${ }^{1}$ This melanoma originates from the uvea, which includes the choroid, the ciliary body, and the iris. ${ }^{2}$ Unlike cutaneous melanoma, UM is not induced by mutations in BRAF, NRAS, or KIT. ${ }^{3}$ Mutations in the G-proteins GNA11 and GNAQ present the driver mutations in uveal melanoma, resulting in activation of the mitogen-activated protein kinases (MAPK) pathway, among others. ${ }^{4,5}$ Recent studies show that the transcriptional coactivator YAP is stimulated by the mutated G-proteins through Trio-Rho/Rac signaling, and that it is an essential factor in mutation-driven uveal melanoma growth. ${ }^{6,7}$ Up to $50 \%$ of patients with uveal melanoma may develop metastases and the prognosis of patients with metastasized tumors is poor: Median survival is approximately 10 to 18 months. ${ }^{8}$ Mutations in the BAP1 gene, encoding a ubiquitin hydrolase, have been associated with the development of metastases. ${ }^{9,10}$ Because of the limited efficacy of current treatments, new therapeutic strategies need to be developed. ${ }^{11}$
Xenotransplantation of uveal melanoma cells has been already successfully applied to immunodeficient mice and rabbits. ${ }^{12,13}$ However, these studies are time consuming and costly. There is a clear need to develop more efficient in vivo models that can be used in preclinical testing and in medium- to highthroughput assays for screening libraries of compounds in drug discovery studies.

The zebrafish may be an excellent model system for this purpose, due to several useful properties. ${ }^{14}$ There are the general benefits such as relatively low housing costs, a large clutch size, ex utero development, transparency, and easy manipulation of the embryo. Specifically focusing on cancer, zebrafish models have the benefit of a high conservation of (proto-)oncogenes and tumor suppressor genes between zebrafish and humans, making the zebrafish model ideal for identifying clinically relevant genes and compounds. ${ }^{15}$ In addition, zebrafish form tumors with similar histopathological and gene-profiling features as human tumors. ${ }^{16}$ Important for our current study, the adaptive immune system in zebrafish 
does not reach maturity until 4 weeks post fertilization, ${ }^{17,18}$ allowing circumvention of graft rejection by using zebrafish in embryonic stages. This has been used previously to permit xenotransplantation with human tumor cells, including cutaneous melanoma cells, without immunosuppression. ${ }^{19-21}$ Moreover, the optical transparency of zebrafish, in combination with the availability of various tissue-specific fluorescent reporter transgenic lines, allows detection of tumor development within 1 week, and offers unique possibilities for high resolution, noninvasive live imaging of fluorescently labeled cancer cells. ${ }^{22-25}$ Furthermore, zebrafish can absorb small molecular weight compounds from water, enabling easy delivery and efficient screening of large numbers of anticancer compounds. ${ }^{26}$

The objective of the present study was to determine whether we can use zebrafish embryos to develop a human uveal melanoma model that allows for rapid, preclinical anticancer drug discovery. A panel of five uveal melanoma cell lines was injected into the yolk of 2-day-old zebrafish embryos, and proliferation and migration was monitored for 6 days. To determine suitability for drug screening, the xenograft model was tested with three different anticancer drugs. We used the second generation dual BCR-ABL/Src inhibitor dasatinib because Src has been reported to be activated in a subset of UM cells. ${ }^{27}$ Furthermore, we used two broad-spectrum cancer inhibitors: quisinostat and MLN-4924. MLN-4924 is an inhibitor of the neddylation pathway, which is essential for the activity of the cullin-RING subtype of the ubiquitin ligases: It blocks turnover of a subset of proteins, resulting in S-phase defects leading to cell apoptosis. $^{28}$ Quisinostat is an experimental pan-inhibitor of histone deacetylase (HDAC) enzymatic activity, currently in phase II trials. $^{29}$

We report that the behavior of the primary and metastatic uveal melanoma cells in this zebrafish xenograft model correlates with their grade of malignancy in humans and that the zebrafish provides a fast, sensitive in vivo vertebrate model for identifying anticancer compounds to combat uveal melanoma metastases.

\section{Materials ANd Methods}

\section{Animal Care and Handling}

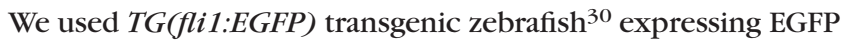
in endothelial cells in a wild-type background; zebrafish were maintained according to standard protocols (http://ZFIN.org, in the public domain), in compliance with the Dutch animal welfare regulations. The research followed the statement or the use of animals in ophthalmic and visual research as published by the Association for Research in Vision and Ophthalmology.

\section{Cell Culture}

Five human UM cell lines were investigated. Two uveal melanoma cell lines derived from primary tumors (92.1, Mel270) and three from UM metastases (OMM1, OMM2.3, OMM2.5). Cell line OMM1 was derived from a uveal melanoma skin metastasis, and cell lines OMM2.3 and -2.5 were obtained from a liver metastasis in the same patient from which cell line Mel270 was derived. Cell line 92.1 was established in the Leiden University Medical Center laboratory, Leiden, The Netherlands. ${ }^{31}$ Cell lines Mel270, OMM2.3, and OMM2.5 came from the Schepens Eye Research Institute, The Massachusetts Eye and Ear Infirmary, Harvard Medical School, Boston ${ }^{32}$; OMM1 cells were kindly provided by G PM Luyten (Rotterdam University Hospital/LUMC, Leiden, The Netherlands). ${ }^{33}$ Cells mentioned above were cultured in 1:1 RPMI-1640 medium + Dulbecco's modified Eagle's medium (DMEM)-F12 (Invitrogen, Carlsbad, CA, USA) with $10 \%$ of fetal calf serum at $37^{\circ} \mathrm{C}$ and $5 \%$ $\mathrm{CO}_{2}$. Human diploid foreskin VH10 fibroblasts were grown in DMEM supplemented with $10 \%$ fetal calf serum. Cell lines were transduced with lentiviral vector CMV-mCherry-Puro (provided by RC Hoeben, LUMC) and selected with puromycin. In short, cells were seeded into 6-well plates 1 day before transduction, at a density of $\sim 3 \times 10^{5}$ cells/well. The next day, cells were transduced $\mathrm{O} / \mathrm{N}$ with the mCherry-expressing lentivirus, with multiplicity of infection $\sim 1.5$, in the presence of $8 \mu \mathrm{g} / \mathrm{mL}$ polybrene. One day later, the virus-containing medium was replaced with fresh growth medium. Selection of transduced cells was started 24 hours later by addition of $0.5 \mu \mathrm{g} / \mathrm{mL}$ puromycin. Transduction of the cells with the mCherry-Puro virus did not noticeably change the growth properties of the cells.

\section{Cell Line Authentication}

Cell line identity was validated by short tandem (STR)-DNA fingerprinting using the AmpF $\ell$ STR Identifier kit according to manufacturer instructions (Applied Biosystems, Carlsbad, CA, USA). We compared the STR profiles to known fingerprints, ${ }^{34}$ which can be found in Supplementary Table $\mathrm{S} 1$.

\section{Western Blotting}

Cells were lysed in Giordano buffer (50 mM Tris-HCl, $\mathrm{pH} 7.4$, $250 \mathrm{mM} \mathrm{NaCl}, 0.1 \%$ Triton X-100, $5 \mathrm{mM}$ EDTA) with proteaseand phosphatase inhibitors. Proteins (15-20 $\mu \mathrm{g}$ total protein lysates) were separated by sodium dodecyl sulfate-polyacrylamide gel electrophoresis, blotted onto commercial membranes (Immobilon-P; Millipore Corp., Billerica, MA, USA), incubated with appropriate primary and secondary antibodies, and bands were visualized by chemoluminescence (West Dura; Pierce Biotechnology, Rockford, IL, USA). Antibodies used were: Anti-p53 (DO-1, 1:500; Santa Cruz Biotechnology, Heidelberg, Germany); Phospho-Src Family (Tyr416) rabbit polyclonal antibody and Src (36D10) rabbit mAb (1:1000; Cell Signaling Technology, Danvers, MA, USA); anti-MAPK, activated, diphosphorylated ERK-1 and 2, (1:1000; Sigma-Aldrich Corp., St. Louis, MO, USA); p44/42 MAPK (ERK1/2; 137F5) rabbit mAb (1:1000; Cell Signaling Technology); anti-cyclin E (HE12, 1:250; Santa Cruz Biotechnology); anti-p27 KIP1 (Y239, 1:1000; Abcam, Cambridge, UK); anti-p21/WAF1/Cip1 (CP74, 1:500; Millipore Corp.); anti-acetyl K9-Histone 3 (cat. \#06-599, 1:2000; Upstate/Millipore Corp.) and anti-Vinculin (hVIN1, 1:1000; Sigma-Aldrich Corp.).

\section{Crystal Violet Staining for Cell Survival}

Cells were seeded in triplicate in 96-well plates: 2500 cells/well in a total volume of $135 \mu \mathrm{L}$ medium. Next day, treatment with compounds was started by adding $15 \mu \mathrm{L}$ of a $\times 10$ concentration. After 72 hours, cell survival was determined by fixating and staining the cells with crystal violet $(0.1 \%)$ for 30 minutes. After rinsing with water, crystal violet was solubilized in $100 \%$ methanol and absorbance (545 nm) was measured in a microplate reader (Victor3 Multilabel Counter 1420-042; Perkin-Elmer, Wellesley, MA, USA).

\section{Human Cancer Cell Implantation}

Before implantation, eggs were kept at $28^{\circ} \mathrm{C}$ and manually dechorionated at 1 day post fertilization (dpf). Cells were grown to $60 \%$ to $90 \%$ confluency, trypsinized (0.25\% trypsin/ $0.53 \mathrm{mM}$ EDTA), centrifuged for 5 minutes at $200 \mathrm{~g}$, washed 

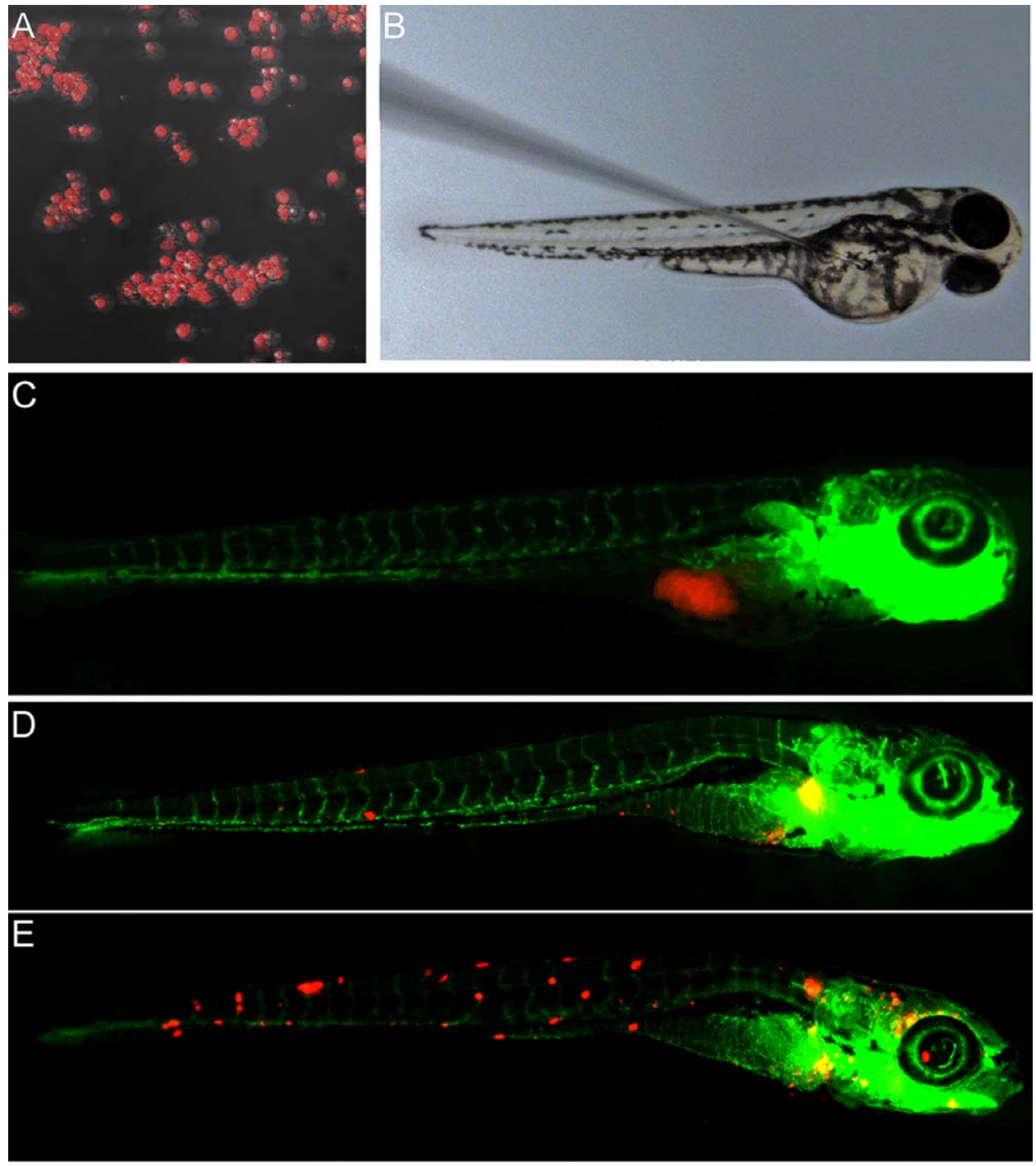

Figure 1. Migration of the uveal melanoma cancer cells outside the yolk of zebrafish embryos. (A) Uveal melanoma cells (OMM1) stably expressing mCherry. (B) Site of injection in a 2-dpf embryo. (C) Embryo 2 dpf TG(fli1:EGFP) with green vasculature, injected with UM cells (OMM1, red), at 1 dpi. At 6 dpi, the injected embryos show active migration of (D) Mel270 and (E) OMM2.3, respectively, in the tail region and in the head. According to these stereo images, the cells can migrate through the host circulation and invade the surrounding tissues. Size and location of tumor masses in 92.1, OMM1 and OMM2.5 cell lines are comparable with OMM2.3. Data are representative stereo fluorescent images (original magnification: $\times 20$ ) of $>10$ independent experiments.

with Dulbecco's phosphate-buffered saline (DPBS; Invitrogen), and taken up in $7 \mu \mathrm{L} 2 \%$ polyvinylpyrrolidone-40 (PVP-40, Calbiochem, San Diego, CA, USA). Cell suspensions were injected using glass capillary needles $(1 \mathrm{~mm}$ OD $\times 0.78 \mathrm{~mm}$ ID; Harvard Apparatus, Holliston, MA, USA) with an approximate opening equal to the dimension of one cell. At 2 dpf, embryos were anesthetized with $0.003 \%$ tricaine (Sigma-Aldrich Corp.) and positioned on a $10-\mathrm{cm}$ Petri dish coated with $1 \%$ agarose. Approximately 400 to 500 cells were injected into the yolk sac, using a pneumatic pico pump and a manipulator (World Precision Instruments, Sarasota, FL, USA). The number of injected cells was determined by counting cells present per droplet of cells after injection on agarose and monitoring the consistency of droplet size after injection in the fish. The number of cells injected was highly consistent, as observed during regular verification of the amount of cells per droplet on agarose. At 1 day post injection (dpi), embryos with cells injected into the circulation were discarded. After the implantation, embryos were kept in egg water $(60 \mu \mathrm{g} / \mathrm{mL}$
OceanSalt in demi water) at $34^{\circ} \mathrm{C}$. This temperature was chosen as an intermediate between $37^{\circ} \mathrm{C}$ (optimal cell temperature) and $28^{\circ} \mathrm{C}$ (optimal fish temperature), which allows for normal development of the embryo ${ }^{20}$ while not impairing UM growth. At 6 dpi, embryos were fixed overnight with $4 \%$ paraformaldehyde (PFA) at $4{ }^{\circ} \mathrm{C}$.

\section{In Vivo Toxicity/Treatment With Chemical Compounds}

The tested compounds were: dasatinib (BMS-354825; Cayman Chemicals, Ann Arbor, MI, USA); quisinostat (JNJ-26481585; Johnson \& Johnson Pharmaceutical R\&D, New Brunswick, NJ, USA); and MLN-4924 (Nedd8-activating enzyme inhibitor; LifeSensors, Malvern, PA, USA).

To test compound toxicity, six 3-dpf embryos were placed per well into a 24 -well plate containing $1 \mathrm{~mL}$ egg water, in which the compound was diluted at different concentrations. The embryos were kept at $34^{\circ} \mathrm{C}$. Medium was refreshed every 
A

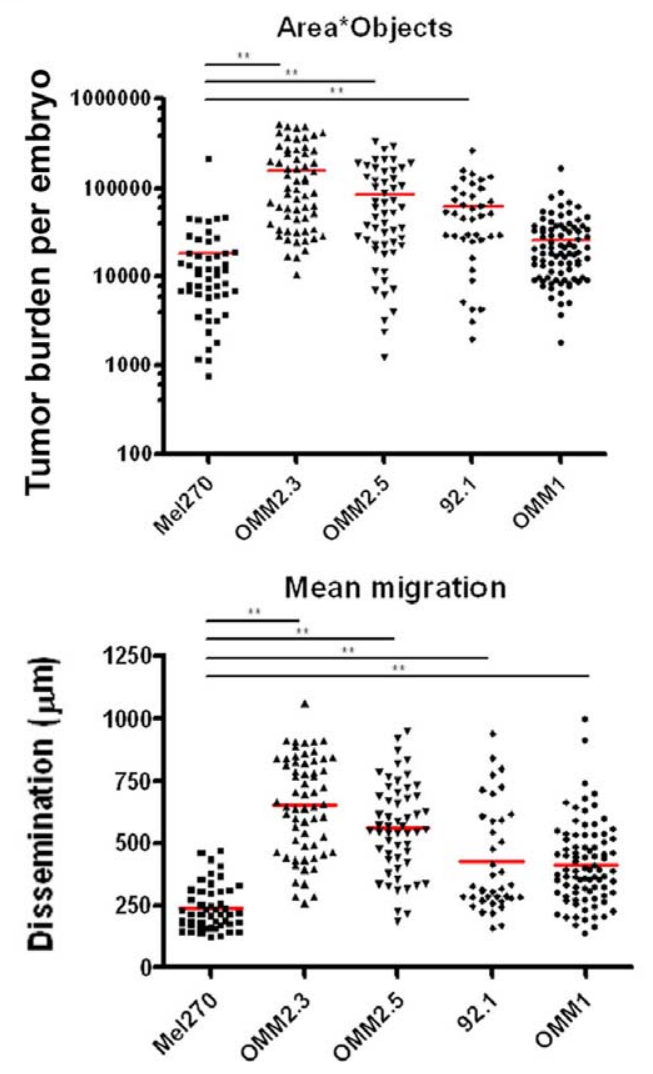

B
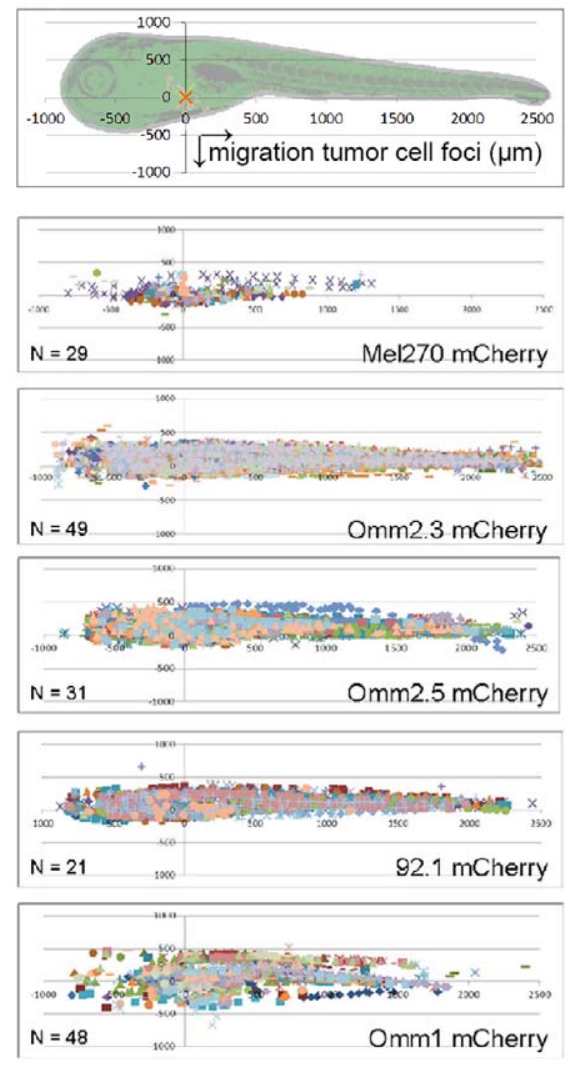

Figure 2. Characterization of migration and proliferation of five UM cell lines in the zebrafish embryonic xenograft model. (A) Quantification of Area*Objects plotted in the logarithmic scale and of mean migration plotted in linear scale. Each filled symbol in the upper panel indicates the tumor burden present per embryo, calculated by multiplying the mean area (in pixels) of all the identified foci with the number of red objects detected. The lower panel indicates the mean distance of migration away from the site of implantation per red object in a single embryo. The data presented are the results of two repeated experiments for each cell line. The number of embryos for each group are $\mathrm{N}_{\text {Mel270 }}=50, \mathrm{~N}_{\mathrm{OMm} 2.3}=61$, $\mathrm{N}_{\text {Омм2.5 }}=55, \mathrm{~N}_{92.1}=39, \mathrm{~N}_{\text {Омм1 } 1}=85$. (B) Scatterplots showing tumor foci dissemination from several embryos injected with each cell line. The upper graph shows the relation between data point coordinates and fish morphology. For each embryo, the calculated injection site, marked with an orange cross, is aligned with the 0,0 coordinates on the graph. Statistical analyses performed with one-way ANOVA test followed by Dunnett's posttest. ${ }^{* *} P<0.01$.

day and survival was checked up to $8 \mathrm{dpf}$. The compound was considered nontoxic for survival rates higher than $80 \%$.

To analyze the effect of treatment, cancer cells were implanted in the yolk sac at $2 \mathrm{dpf}$. At $1 \mathrm{dpi}$, treatment was started on implanted embryos, with experimental conditions identical to the toxicity tests. Embryos at 6 dpi were fixed with $4 \%$ PFA and stored at $4^{\circ} \mathrm{C}$.

\section{Microscopy and Image Analysis}

Stereo images were taken with a fluorescence stereo microscope (Leica M205FA; Leica Microsystems, Inc., Buffalo Grove, IL, USA). Confocal images were acquired using a confocal microscope (Leica Microsystems, Inc., or NIKON3, $\times 4$ lens; NIKON Corp., Tokyo, Japan). Live embryos were anesthetized with $0.003 \%$ tricaine (Sigma-Aldrich Corp.) and kept in egg water. Fixed embryos were embedded in a layer of $1.5 \%$ methyl cellulose diluted in egg water, $1 \%$ low melting agarose or $0.1 \%$ PBS-T, and imaged in glass-bottom 96-well plates. Image processing was performed with ImageJ 1.43 (http://imagej. nih.gov/ij/; provided in the public domain by the National Institutes of Health, Bethesda, MD, USA). Image analysis (ImagePro Analyzer 7.0; Media Cybernetics, Rockville, MD, USA) was performed with a macro developed in our laboratory, as described previously. ${ }^{35}$ In brief, this macro is able to detect each individual red fluorescent cluster inside the embryo and quantify its distance from the site of implantation. The mean distance from the site of implantation for each tumor cell cluster was used to measure the migration of cancer cells in a single embryo (mean migration). Additionally, the mean proliferation readout was estimated per embryo by the multiplication of the mean area of all tumor foci with the total number of red objects (Area*Objects).

\section{Statistics}

Statistical analysis was performed using graphing and statistical software (GraphPad PRISM, version 4.0; GraphPad, San Diego, CA, USA). Differences between two or more means were determined using Student's unpaired $t$-test and one-way ANOVA with Dunnett's posttest. Statistical significance was indicated in the following way: ${ }^{*} P<0.05,{ }^{* *} P<0.01$, and ${ }^{* * * *} P$ $<0.001$.

\section{RESUlts}

\section{Active Migration of UM Cells in Zebrafish Embryos}

To determine if the zebrafish is a suitable organism for modeling UM tumor progression via engraftment, we selected 

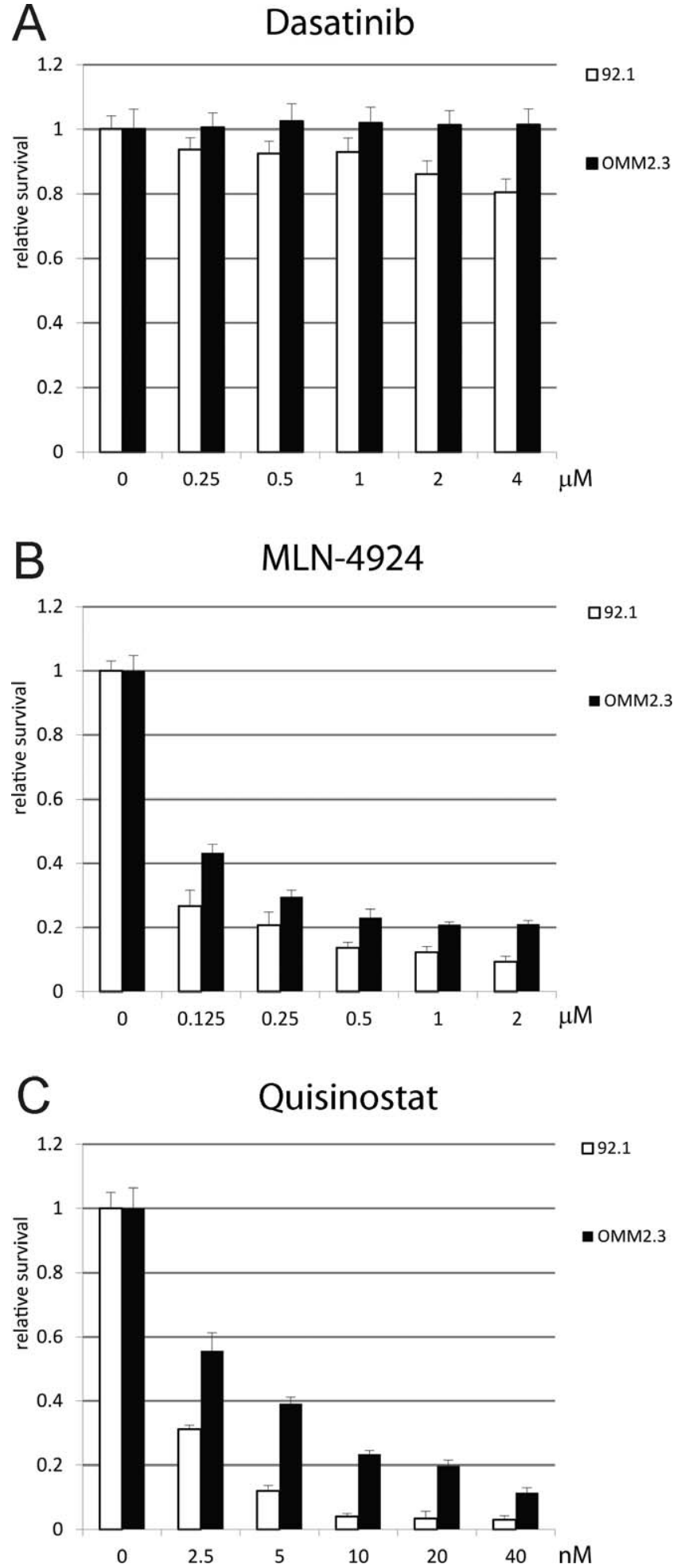

Figure 3. Growth inhibition of UM cells in cell culture. We used mCherry-expressing cell lines Mel270, OMM2.3, and 92.1 in a proliferation assay as described in the "Materials and Methods" section. Cells were treated for 72 hours with indicated concentrations of (A) dasatinib, (B) MLN-4924, and (C) quisinostat.

five UM cell lines, labeled them with red fluorescent mCherry (Fig. 1A), and implanted 400 to 500 labeled cells into the yolk of 2-dpf TG(fli1:EGFP) embryos with green fluorescent vessels (Fig. 1B). Embryos were screened at $1 \mathrm{dpi}$ for successful implantation ( $>90 \%$ for each cell line in every experiment; Fig. 1C). Hematogenous dissemination to the head and tail region of the zebrafish larvae was detected for all tested cell lines from 4 dpi onward. The cancer cell dissemination was considered as active migration if the cells were beyond the boundaries with the heart cavity frontally, on top of the swim bladder dorsally and beyond the urogenital opening caudally. Migration of cells out of the yolk was first observed at $4 \mathrm{dpi}$, and increased at 5 and $6 \mathrm{dpi}$, at which time approximately $50 \%$ of the total amount of injected embryos had migrating cells. At $6 \mathrm{dpi}$, a variation in the amount of cell burden was observed for different cell lines (Figs. 1D, 1E). Importantly, the primary tumor-derived Mel270 (Fig. 1D) showed less dissemination then metastasis-derived OMM2.3 (Fig. 1E). The human foreskin fibroblasts VH10 did not migrate from the yolk sac (data not shown). In a previous study, we used $3 \mathrm{~T} 3$ cells, ${ }^{24}$ and normal melanocytes were previously described as less migratory in comparison with human metastatic melanoma, ${ }^{19}$ suggesting that cell dissemination in zebrafish embryo is cancer cellspecific.

\section{Differential Migration of Primary and Metastatic UM Cells in the Zebrafish Xenograft Model}

In order to quantify the behavior of different UM cell lines in zebrafish embryos, an automated whole animal bioimaging assay was applied to confocal microscopy images of fixed $6 \mathrm{dpi}$ embryos. For quantitative measurements of cell proliferation and migration, $z$-stack images were analyzed using a macro (Media Cybernetics) previously developed in our laboratory. ${ }^{35}$ Readout of this analysis is seen in Figure 2. All uveal melanoma cell lines showed migration and proliferation, but differences between cell lines were observed: primary tumor cell line Mel270 proliferated and migrated significantly less compared with metastatic OMM2.3 and OMM2.5 cells, derived from metastases from the same patient (Figs. 2A, 2B). The tumor burden induced by Mel270 was significantly lower than the burden from primary tumor cell line 92.1. In addition, the metastatic OMM1 cell line migrated more than Mel270, while proliferation was only slightly, and not significantly, higher than that of Mel270 (Figs. 2A, 2B).

The scatterplots (Fig. 2B) indicate the localization of all cancer foci relative to the site of implantation $(0 ; 0)$, using a different color for each embryo analyzed. The Mel270 scatterplot shows only a few cancer foci which are mainly clustered close to the yolk area, whereas the other cell lines, OMM2.3 in particular, are disseminated all through the head region, the trunk, and the tail.

All together, the results in the embryonic zebrafish engraftment model are consistent with the clinical behavior of uveal melanoma in human.

\section{Effects of Dasatinib, Quisinostat, and MLN-4924 In Vitro}

Metastatic OMM2.3 and primary 92.1 UM cell lines in cell culture were exposed to various concentrations of dasatinib, an Src tyrosine kinase inhibitor, since previous experiments showed activation of Src in a subset of UM cell lines. ${ }^{27}$ In addition, we used two experimental anticancer drugs: MLN4924 and quisinostat.

An in vitro 72-hour treatment with $4 \mu \mathrm{M}$ dasatinib inhibited growth of cell line 92.1 by $20 \%$ (Fig. 3A). Incubation with MLN4924 or quisinostat resulted in strong growth inhibition of both cell lines, even at the lowest concentration tested, $0.125 \mu \mathrm{M}$ and $2.5 \mathrm{nM}$, respectively (Figs. 3B, 3C). Anticancer drug MLN$4924(1 \mu \mathrm{M})$ reduced survival of OMM2.3 and 92.1 cells to approximately $20 \%$ (Fig. 3B). The efficacy of quisinostat was 

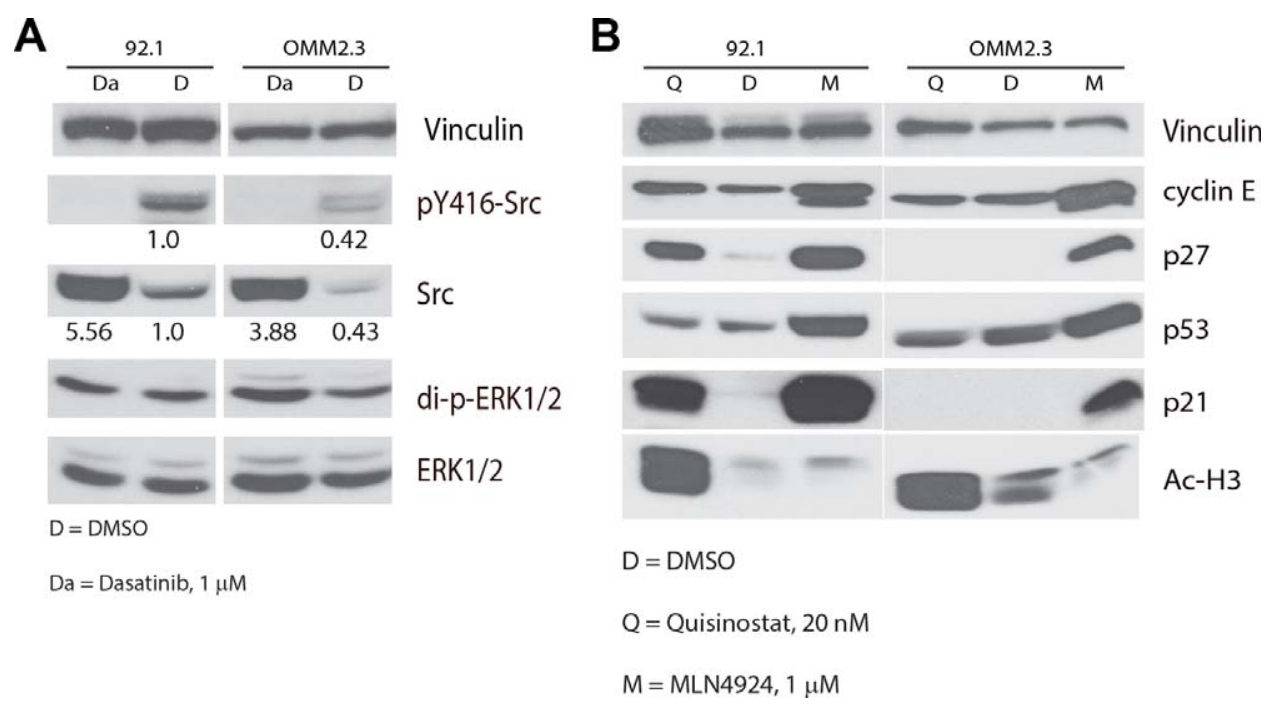

FiguRE 4. Western blot analysis of proteins from uveal melanoma lines exposed to anticancer drugs (A) 92.1/ and OMM2.3/mCherry cells were either dimethylsulfoxide (DMSO)-treated (D) or treated with $1 \mu \mathrm{M}$ dasatinib (Da) for 24 hours. The levels and phosphorylation of Src and ERK1/2 were determined by Western blot analysis. Quantification of Src-levels is indicated underneath the blots. (B) 92.1 and OMM2.3/mCherry cells were either DMSO-treated (D) or treated with $20 \mathrm{nM}$ quisinostat (Q) or with $1 \mu \mathrm{M}$ MLN-4924 (M) for 24 hours. Expression of a number of published targets of MLN-4924 and quisinostat is shown.

even more dramatic. At a 10-nM concentration, this drug completely blocked proliferation of 92.1 and inhibited growth of OMM2.3 by $80 \%$ (Fig. 3C).

Western blot analyses indicated higher basal- and pY416-Src levels in the untreated 92.1 than in OMM2.3 cells; quantification indicated a $\sim 2.5$-fold higher level in 92.1 cells. Treatment with dasatinib efficiently blocked the Y416 phosphorylation level of Src in both cell lines (Fig. 4A). Interestingly, total levels of Src increased upon dasatinib treatment. This effect is likely caused by distinct ubiquitination and degradation of Tyr416phosphorylated versus nonphosphorylated Src protein, as has been published by Harris et al. ${ }^{36}$

In addition, the protein levels of ERK1/2 and phosphorylated ERK1/2 were the same in both cell lines and remained unchanged after dasatinib treatment.

Figure $4 \mathrm{~B}$ shows the target proteins affected in 92.1 and OMM2.3 cells after treatment with quisinostat or MLN-4924. Interestingly, quisinostat only detectably increased p21 and p27 levels in 92.1 cells while MLN-4924 elevated p21, p27, and p53 in both cell types. It should be noted that basal levels of p21 and p27 are almost undetectable in OMM2.3 cells. In addition, as expected, quisinostat treatment increased the acetylation of K9-histone 3 in both cell lines. All together, these data showed that the inhibitors blocked UM cell survival with distinct activities and via distinct mechanisms.

\section{Toxicity of Dasatinib, Quisinostat, and MLN-4924}

The next step was to validate the zebrafish embryonic xenograft model as described above by analyzing the tumor response to anticancer drug treatments in vivo. To determine which concentrations of dasatinib, MLN-4924, and quisinostat would be well tolerated-with minimal effects on development and survival-noninjected embryos were immersed in these compounds, a well-established method for drug treatment of zebrafish larvae. ${ }^{26,37}$

Dasatinib was found to be toxic after 4 to 5 days' exposure at concentrations higher than $1 \mu \mathrm{M}$ (Fig. 5A). Neither quisinostat (Fig. 5B) nor MLN-4924 (Fig. 5C) was toxic to the embryos, even at the highest concentration tested $(1 \mu \mathrm{M})$.

\section{Effects of Dasatinib, Quisinostat, and MLN-4924 on UM Xenografts}

After determining the optimal concentration for each chemical inhibitor, treatments were performed on injected embryos. Two cell lines were chosen for these experiments: metastatic OMM2.3, as it was the most aggressive cell line in the zebrafish embryonic xenograft model, and primary tumor cell line 92.1. Mel270 cells were not used since basal proliferation and migration of these cells in untreated embryos were quite low (Fig. 2).

Embryos injected with OMM2.3- or 92.1-mCherry cells at 2 dpf were treated at $1 \mathrm{dpi}$ with $1 \mu \mathrm{M}$ dasatinib, and showed a significantly reduced growth of 92.1 (mean reduction with a $95 \%$ confidence interval $[\mathrm{MR}]$ was $55.0 \% \pm 25.2 \%, P<0.05)$, while growth of OMM2.3 cells was not affected (Fig. 6). The distance of migration from the site of implantation indicates that treatment with dasatinib significantly reduced dissemination of 92.1 cells $\left(M_{\text {migration }}=29.9 \% \pm 8.5 \%\right)$ and not of OMM2.3.

The treatment of injected embryos with the broad-spectrum anticancer drugs MLN-4924 and quisinostat significantly inhibited both the proliferation and migration of OMM2.3 and 92.1 cells. For anticancer drug MLN-4924, OMM2.3 mean reductions were as follows: $\mathrm{MR}_{\text {growth }}=46.5 \% \pm 15.4 \%$ and $\mathrm{MR}_{\text {migration }}=13.9 \% \pm 5.5 \%$. Compared with OMM2.3, MLN4924 inhibited 92.1 growth and migration slightly more: $\mathrm{MR}_{\text {growth }}=55.9 \% \pm 15.9 \%$ and $\mathrm{MR}_{\text {migration }}=25.1 \% \pm 7.3 \%$. Quisinostat was able to inhibit both OMM2.3 (MR growth $=$ $\left.45.6 \% \pm 16.3 \%, \mathrm{MR}_{\text {migration }}=13.7 \% \pm 5.5 \%\right)$ and 92.1 cells $\left(\mathrm{MR}_{\text {growth }}=50.6 \% \pm 15.6 \%, \mathrm{MR}_{\text {migration }}=18.1 \% \pm 7.3 \%\right)$. The 92.1 cells were again slightly more sensitive to quisinostat than the metastatic OMM2.3 cells, as also seen in the cell culture (Figs. 3, 7A, 7B). These two drugs seem to have a wider effect on cancer proliferation compared with the dissemination of cancer cells in the embryo.

Our results demonstrate that in vivo drug treatment of uveal melanoma-containing zebrafish inhibited the proliferation of these tumor cells as observed in vitro, and also reduced the migration of the UM cells in vivo. However, the zebrafish model has the distinct advantage of determining the effects of 


\section{A Dasatinib}

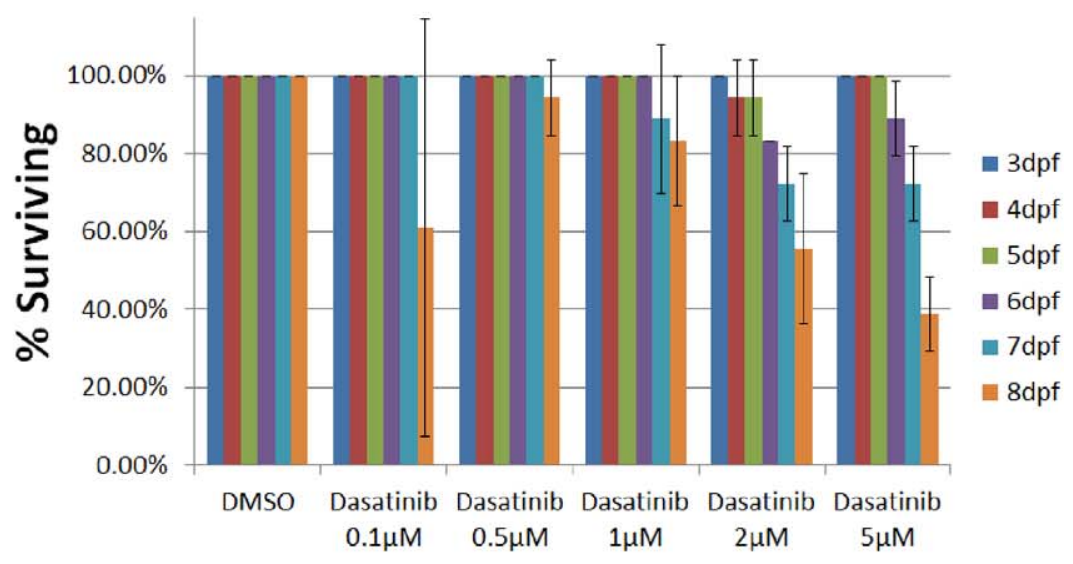

B

\section{MLN-4924}

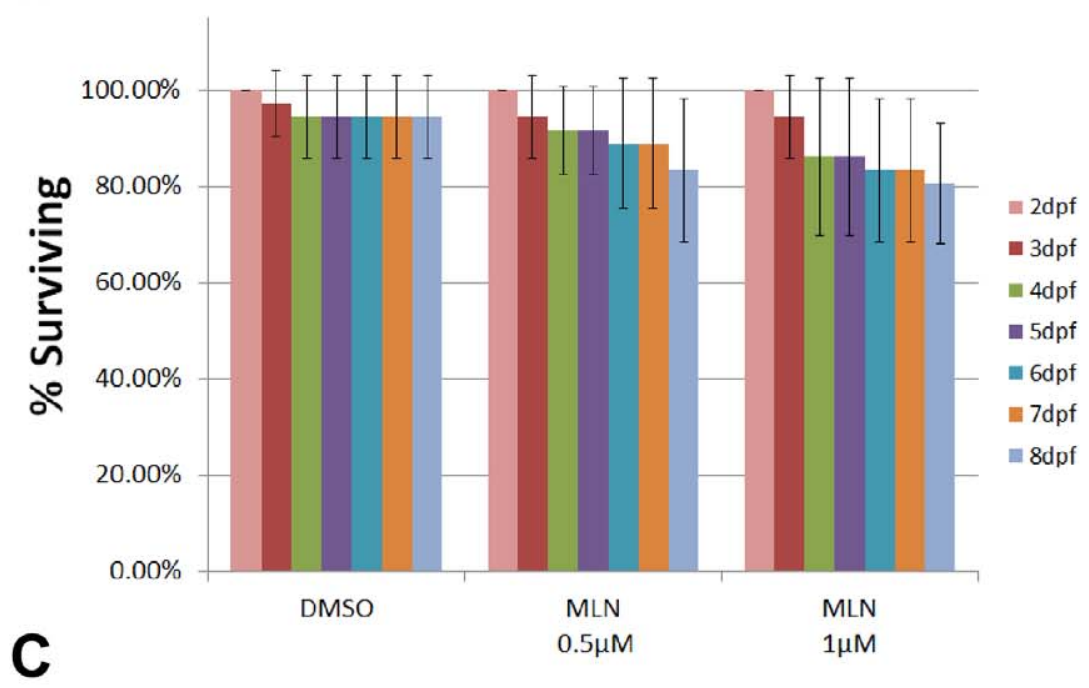

\section{Quisinostat}

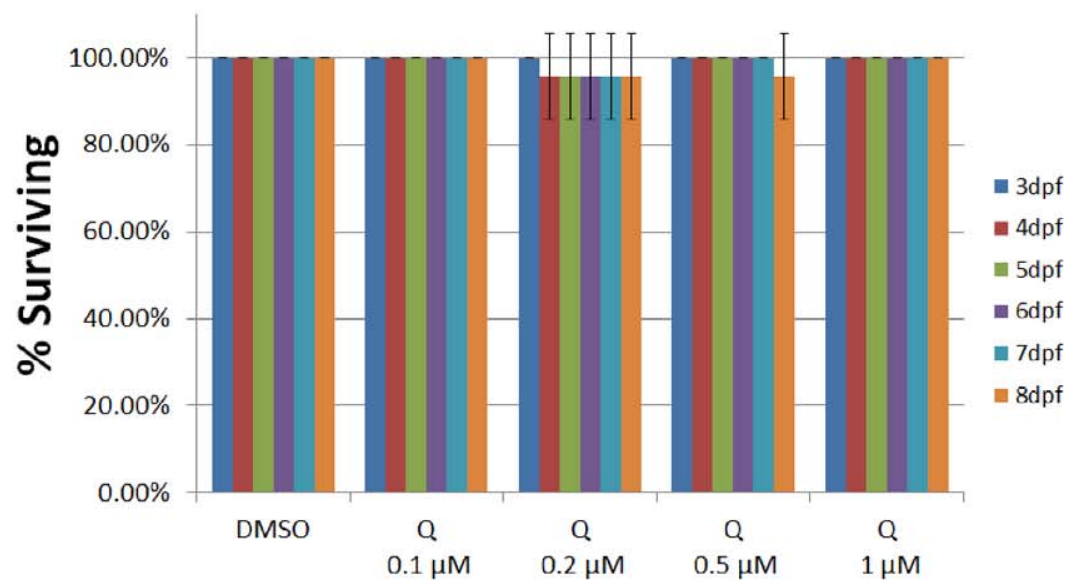

Figure 5. Survival of embryos during anticancer compound toxicity testing. Uninjected 3-dpf embryos were placed in 24-well plates exposed to various concentrations of (A) dasatinib, (B) MLN-4924, and (C) quisinostat and their survival was examined until 8 dpf. $n=24$; error bars show standard error of the mean. 


\section{$1 \mu \mathrm{M}$ Dasatinib}

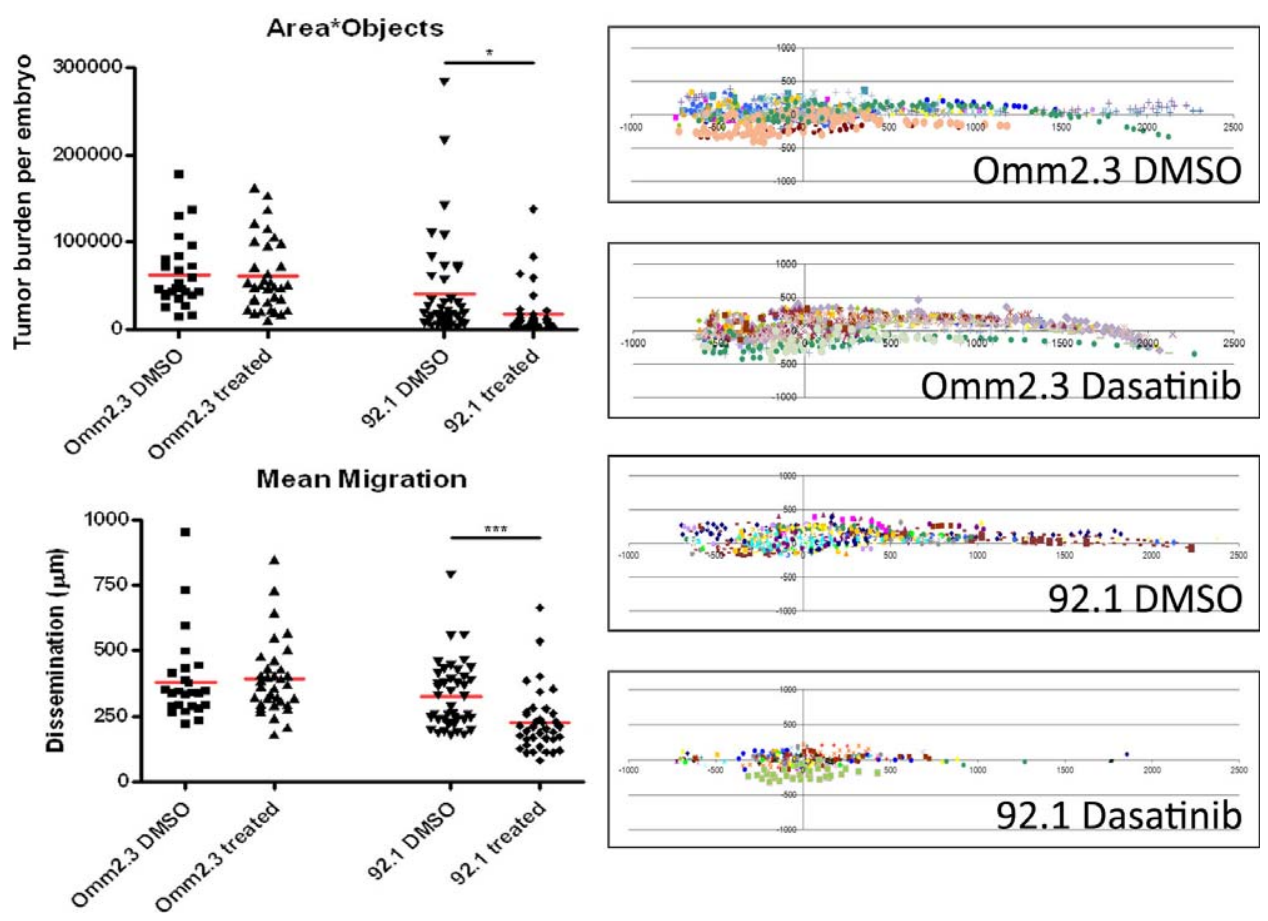

FIGURE 6. Plots representing area*objects and mean migration of OMM2.3 and 92.1 injected embryos treated with $1 \mu \mathrm{M}$ dasatinib or DMSO solvent at 6 dpi. The number of embryos are: for the OMM2.3 groups: $\mathrm{N}_{\text {DMSO }}=26, \mathrm{~N}_{\text {dasatinib }}=34$; and for the 92.1 groups: $\mathrm{N}_{\mathrm{DMSO}}=44$ and $\mathrm{N}_{\text {dasatinib }}=37$. Scatterplots in the right panel visualizing the same data presented in the left panel. Statistical analyses performed with Student's $t$-test. ${ }^{*} P<0.05$. ${ }^{* *} P<0.01$. ${ }^{* * *} P<0.001$.

drug treatment on not only the signaling pathways, but also on tumor growth and metastatic spread, which is not possible using in vitro screening assays.

\section{Discussion}

The prognosis of patients diagnosed with metastatic uveal melanoma is poor and an effective therapeutic strategy is lacking. A number of different investigators have used a variety of immunodeficient mouse strains to develop human-to-mouse xenograft tumor models to study uveal melanoma. ${ }^{38-42}$ Tumor growth was reported within the anterior chamber and vitreous cavity, with at least one report of metastatic spread of tumors from the eye to the liver. Investigators have also used direct injection of tumor cells into the liver to model metastatic liver tumors. These human uveal melanoma tumor models have been extremely useful in studies that identify the unique gene mutations required for malignant transformation of uveal melanoma. For example, RNAi-mediated depletion of BAP1 in uveal melanoma cells resulted in reduced tumor growth in vivo $^{43}$ and expression of the GNAQ ${ }^{\mathrm{Q} 209 \mathrm{~L}}$ mutation in normal melanocytes triggered uncontrolled proliferation and malignant transformation in vivo. ${ }^{44}$ While these mouse tumor models have been very useful to study specific gene pathways, they have limited utility for large scale anticancer drug screening. The most important limitations are: slow tumor growth and spread, which can take weeks to months; variation in tumor growth rate; the high cost of breeding and housing large numbers of mice; and, while tumor growth can be quantitated in vivo in mice, it is time consuming when examining large cohorts of mice.

To facilitate the identification of promising new compounds for treatment of uveal melanoma patients, we describe here the establishment of a novel uveal melanoma zebrafish engraftment model. In recent years, it has been shown that xenograft models in zebrafish embryo allow time-effective, dynamic quantification of tumor burden in vivo. The transparency of the embryos makes them ideal for noninvasive, high-end microscopy, providing the possibility to follow processes such as angiogenesis and leukocyte infiltration as the tumor progresses. Additionally, automated imaging platforms and analysis software has been developed, permitting rapid imaging of tumor progression in a large number of embryos. Furthermore, compounds can be taken up from the water in which the embryos reside, facilitating screening of single and combined compounds. Such screening can be done for multiple xenografted UM cell lines, reflecting the genetic variability of this cancer and the subsequent differential responses to drugs.

After implantation in the yolk sac of $2 \mathrm{dpf}$ zebrafish embryos, engrafted uveal melanoma cells successfully migrated within the embryo body from 4 dpi onward. The automated quantitative analysis of low-magnification confocal laser scanning images of 6 dpi fixed embryos revealed phenotypic differences between UM cell lines that are not evident from cell culture analysis, with metastatic cell lines behaving more aggressively in the zebrafish larvae. As these differences may be correlated with the poor prognosis of UM patients, we evaluated treatment efficacy of three drugs in both a primary and a metastatic cell line.

The effect of dasatinib was more pronounced with regard to migration than proliferation of engrafted 92.1 cells, and suggests that inhibition of Src phosphorylation mainly controls cell motility. In both 92.1 and OMM2.3, dasatinib inhibits Src phosphorylation in an ERK1/2-independent manner. The resistance of OMM2.3 cells to dasatinib is most likely caused 
A

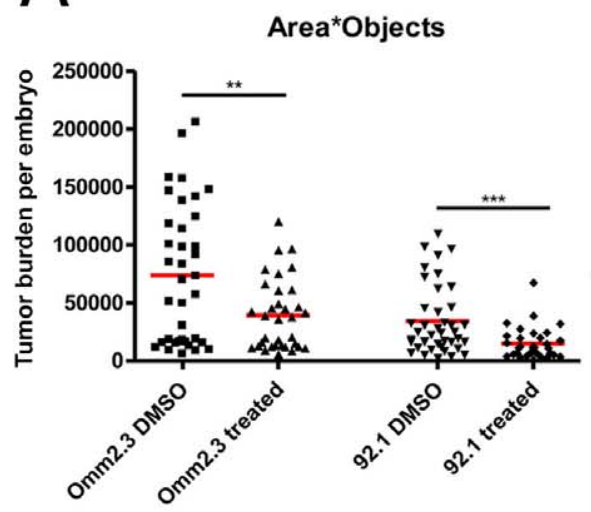

Mean Migration

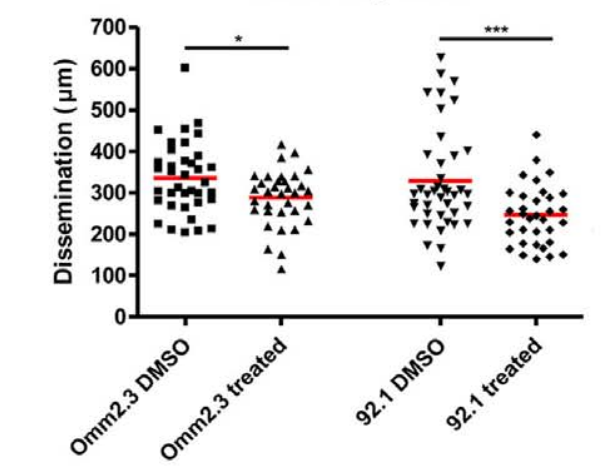

B

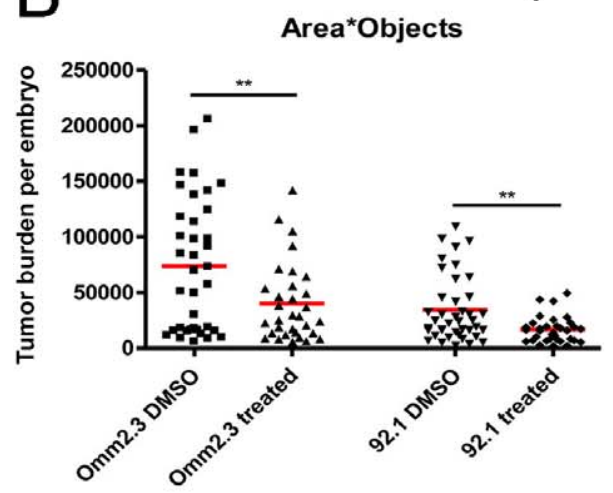

Mean Migration

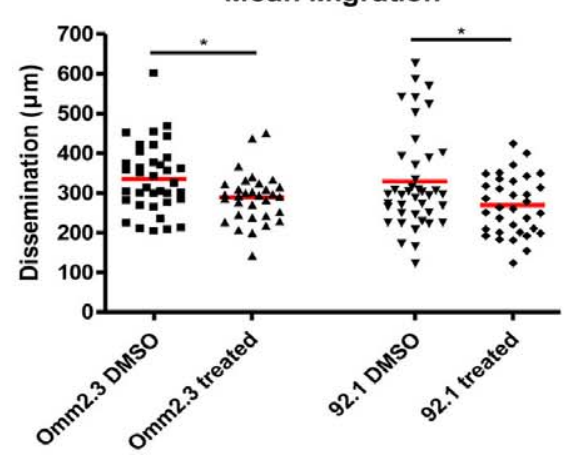

$1 \mu \mathrm{M}$ MLN-4924
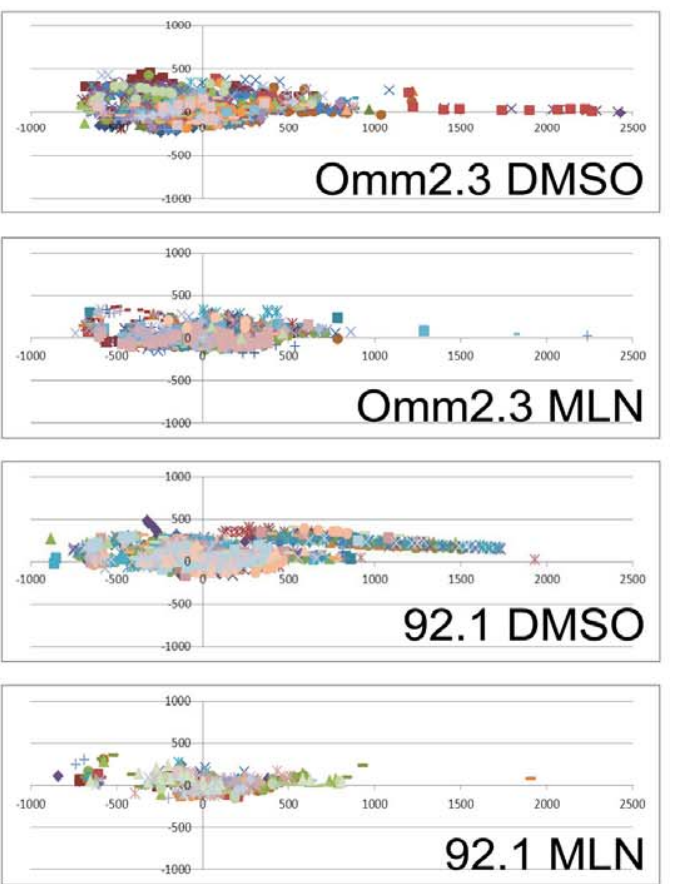
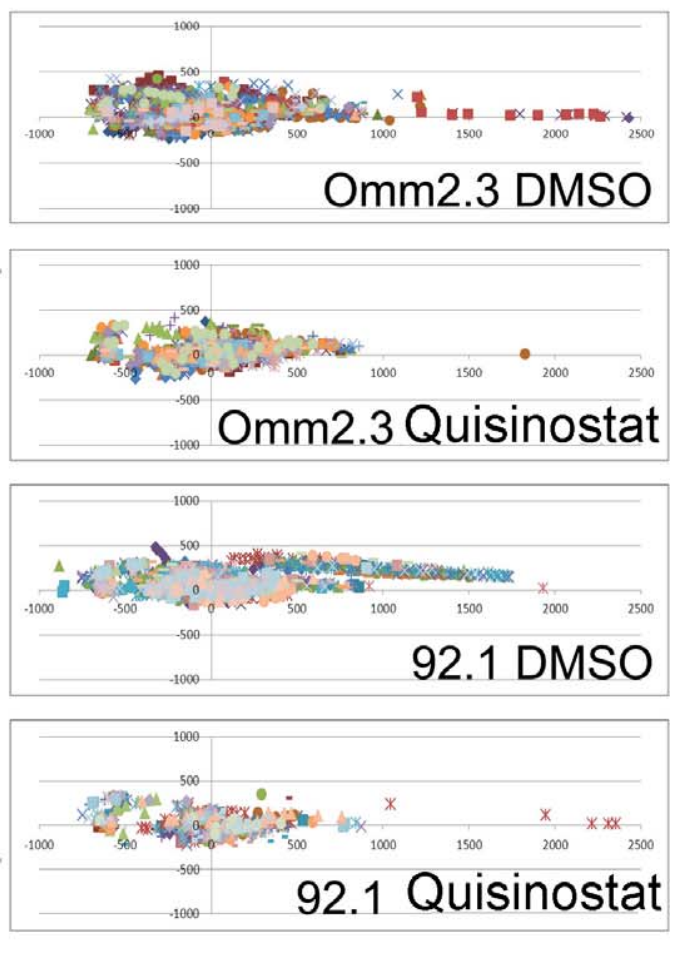

FiguRE 7. Characterization of proliferation and migration of OMM2.3- and 92.1-injected embryos treated with two broad-spectrum cancer inhibitors: quisinostat and MLN-4924 at 6 dpi. The number of embryos for the OMM2.3 groups: $\mathrm{N}_{\mathrm{DMSO}}=37, \mathrm{~N}_{\mathrm{Q}}=32 ;$ for the 92.1 groups: $\mathrm{N}_{\mathrm{DMs}}=$ $41, \mathrm{~N}_{\mathrm{Q}}=34$, and for the OMM2.3 groups: $\mathrm{N}_{\mathrm{DMSO}}=37, \mathrm{~N}_{\mathrm{MLN}}=34$. Scatterplots in the right panel visualize the same data as presented in the left panel. Statistical analyses performed with Student's $t$-test. ${ }^{*} P<0.05$. ${ }^{* *} P<0.01$. ${ }^{* * * *} P<0.001$. 
by the lower level of Src in this metastasis-derived cell line (Fig. $4 \mathrm{~A}$ and Maat et $\mathrm{al}^{27}$ ).

The administration of the broad-spectrum cancer inhibitors MLN-4924 and quisinostat to engrafted embryos strongly inhibited both proliferation and migration of both tested cell lines. The primary 92.1 cells were more sensitive to MLN-4924 and quisinostat treatment than metastatic OMM2.3 cells. This was also observed in culture, and suggests that inhibitor treatment in vivo correlates with treatment in culture.

Although some differences in response regarding upregulation of p21, p27, and p53 are noticed between these two drugs and the two tested cell lines, the exact mechanism(s) by which these drugs affect cell cycle progression and survival are complex and not fully understood. However, because of the results described here, further investigations on the possible use of Quisinostat and MLN-4924 for uveal melanoma patients is warranted.

In summary, this study shows that the here described zebrafish xenograft model can be used for preclinical analysis of new anticancer compounds against uveal melanoma in a time- and cost-effective manner.

\section{Acknowledgments}

Supported by Stichting Kinderen Kankervrij, Project 30677.

Disclosure: W. van der Ent, None; C. Burrello, None; A.F.A.S. Teunisse, None; B.R. Ksander, None; P.A. van der Velden, None; M.J. Jager, None; A.G. Jochemsen, None; B.E. SnaarJagalska, None

\section{References}

1. Egan KM, Seddon JM, Glynn RJ, Gragoudas ES, Albert DM. Epidemiologic aspects of uveal melanoma. Surv Ophthalmol. 1988;32:239-251.

2. Singh AD, Bergman L, Seregard S. Uveal melanoma: epidemiologic aspects. Ophthalmol Clin North Am. 2005;18:75-84, viii.

3. Zuidervaart W, van Nieuwpoort F, Stark M, et al. Activation of the MAPK pathway is a common event in uveal melanomas although it rarely occurs through mutation of BRAF or RAS. $\mathrm{Br}$ J Cancer. 2005;92:2032-2038.

4. Van Raamsdonk CD, Bezrookove V, Green G, et al. Frequent somatic mutations of GNAQ in uveal melanoma and blue naevi. Nature. 2009;457:599-602.

5. Van Raamsdonk CD, Griewank KG, Crosby MB, et al. Mutations in GNA11 in uveal melanoma. $N$ Engl $J$ Med. 2010;363:2191-2199.

6. Yu FX, Luo J, Mo JS, et al. Mutant Gq/11 promote uveal melanoma tumorigenesis by activating YAP. Cancer Cell. 2014; 25:822-830.

7. Feng X, Degese MS, Iglesias-Bartolome R, et al. Hippoindependent activation of YAP by the GNAQ uveal melanoma oncogene through a trio-regulated rho GTPase signaling circuitry. Cancer Cell. 2014;25:831-845.

8. Augsburger JJ, Correa ZM, Shaikh AH. Effectiveness of treatments for metastatic uveal melanoma. Am J Ophthalmol. 2009;148:119-127.

9. Harbour JW, Onken MD, Roberson ED, et al. Frequent mutation of BAP1 in metastasizing uveal melanomas. Science. 2010;330:1410-1413.

10. Wiesner T, Obenauf AC, Murali R, et al. Germline mutations in BAP1 predispose to melanocytic tumors. Nat Genet. 2011;43: 1018-1021.

11. Damato B. Progress in the management of patients with uveal melanoma. The 2012 Ashton Lecture. Eye (Lond). 2012;26: 1157-1172.
12. Ozerdem U, Mach-Hofacre B, Varki N, et al. The effect of prinomastat (AG3340), a synthetic inhibitor of matrix metalloproteinases, on uveal melanoma rabbit model. Curr Eye Res. 2002;24:86-91.

13. Ly LV, Baghat A, Versluis M, et al. In aged mice, outgrowth of intraocular melanoma depends on proangiogenic M2-type macrophages. J Immunol. 2010;185:3481-3488.

14. Goessling W, North TE, Zon LI. New waves of discovery: modeling cancer in zebrafish. J Clin Oncol. 2007;25:24732479.

15. Howe K, Clark MD, Torroja CF, et al. The zebrafish reference genome sequence and its relationship to the human genome. Nature. 2013;496:498-503.

16. Amatruda JF, Shepard JL, Stern HM, Zon LI. Zebrafish as a cancer model system. Cancer Cell. 2002;1:229-231.

17. Lieschke GJ, Trede NS. Fish immunology. Curr Biol. 2009;19: R678-R682.

18. Lam SH, Chua HL, Gong Z, Lam TJ, Sin YM. Development and maturation of the immune system in zebrafish, Danio rerio: a gene expression profiling, in situ hybridization and immunological study. Dev Comp Immunol. 2004;28:9-28.

19. Lee LM, Seftor EA, Bonde G, Cornell RA, Hendrix MJ. The fate of human malignant melanoma cells transplanted into zebrafish embryos: assessment of migration and cell division in the absence of tumor formation. Dev Dyn. 2005;233:1560-1570.

20. Haldi M, Ton C, Seng WL, McGrath P. Human melanoma cells transplanted into zebrafish proliferate, migrate, produce melanin, form masses and stimulate angiogenesis in zebrafish. Angiogenesis. 2006;9:139-151.

21. Konantz M, Balci TB, Hartwig UF, et al. Zebrafish xenografts as a tool for in vivo studies on human cancer. Ann N Y Acad Sci. 2012;1266:124-137.

22. Stoletov K, Klemke R. Catch of the day: zebrafish as a human cancer model. Oncogene. 2008;27:4509-4520.

23. He S, Lamers GE, Beenakker JW, et al. Neutrophil-mediated experimental metastasis is enhanced by VEGFR inhibition in a zebrafish xenograft model. J Pathol. 2012;227:431-445.

24. Drabsch Y, He S, Zhang L, Snaar-Jagalska BE, Ten Dijke P. Transforming growth factor-beta signalling controls human breast cancer metastasis in a zebrafish xenograft model. Breast Cancer Res. 2013;15:R106.

25. van der Ent W, Jochemsen AG, Teunisse AF, et al. Ewing sarcoma inhibition by disruption of EWSR1-FLI1 transcriptional activity and reactivation of p53. J Pathol. 2014;233:415-424.

26. Zon LI, Peterson RT. In vivo drug discovery in the zebrafish. Nat Rev Drug Discov. 2005;4:35-44.

27. Maat W, el Filali M, Dirks-Mulder A, et al. Episodic Src activation in uveal melanoma revealed by kinase activity profiling. Br J Cancer. 2009;101:312-319.

28. Soucy TA, Smith PG, Milhollen MA, et al. An inhibitor of NEDD8-activating enzyme as a new approach to treat cancer. Nature. 2009; 458:732-736.

29. Arts J, King P, Marien A, et al. JNJ-26481585, a novel "secondgeneration" oral histone deacetylase inhibitor, shows broadspectrum preclinical antitumoral activity. Clin Cancer Res. 2009; 15:6841-6851.

30. Lawson ND, Weinstein BM. In vivo imaging of embryonic vascular development using transgenic zebrafish. Dev Biol. 2002;248:307-318.

31. De Waard-Siebinga I, Blom DJ, Griffioen M, et al. Establishment and characterization of an uveal-melanoma cell line. Int $J$ Cancer. 1995;62:155-161.

32. Chen PW, Murray TG, Uno T, Salgaller ML, Reddy R, Ksander BR. Expression of MAGE genes in ocular melanoma during progression from primary to metastatic disease. Clinical Exp Metastasis. 1997; 15:509-518.

33. Luyten GP, Naus NC, Mooy CM, et al. Establishment and characterization of primary and metastatic uveal melanoma cell lines. Int J Cancer. 1996;66:380-387. 
34. Griewank KG, Yu X, Khalili J, et al. Genetic and molecular characterization of uveal melanoma cell lines. Pigment Cell Melanoma Res. 2012;25:182-187.

35. Ghotra VP, He S, de Bont H, et al. Automated whole animal bioimaging assay for human cancer dissemination. PLoS One. 2012;7:e31281.

36. Harris KF, Shoji I, Cooper EM, Kumar S, Oda H, Howley PM. Ubiquitin-mediated degradation of active Src tyrosine kinase. Proc Natl Acad Sci U S A. 1999;96:13738-13743.

37. Peterson RT, Macrae CA. Systematic approaches to toxicology in the zebrafish. Annu Rev Pharmacol Toxicol. 2012;52:433453.

38. Kan-Mitchell J, Mitchell MS, Rao N, Liggett PE. Characterization of uveal melanoma cell lines that grow as xenografts in rabbit eyes. Invest Ophthalmol Vis Sci. 1989;30:829-834.

39. Heegaard S, Spang-Thomsen M, Prause JU. Establishment and characterization of human uveal malignant melanoma xenografts in nude mice. Melanoma Res. 2003;13:247-251.
40. Yang H, Fang G, Huang X, Yu J, Hsieh CL, Grossniklaus HE. Invivo xenograft murine human uveal melanoma model develops hepatic micrometastases. Melanoma Res. 2008;18:95103 .

41. Nemati F, Sastre-Garau X, Laurent C, et al. Establishment and characterization of a panel of human uveal melanoma xenografts derived from primary and/or metastatic tumors. Clin Cancer Res. 2010;16:2352-2362.

42. Braun RD, Vistisen KS. Modeling human choroidal melanoma xenograft growth in immunocompromised rodents to assess treatment efficacy. Invest Ophthalmol Vis Sci. 2012;53:26932701.

43. Matatall KA, Agapova OA, Onken MD, Worley LA, Bowcock AM, Harbour JW. BAP1 deficiency causes loss of melanocytic cell identity in uveal melanoma. BMC Cancer. 2013;13:371.

44. Chen X, Wu Q, Tan $\mathrm{L}$, et al. Combined PKC and MEK inhibition in uveal melanoma with GNAQ and GNA11 mutations [published online ahead of print October 23, 2013]. Oncogene. doi:10.1038/onc.2013.418. 